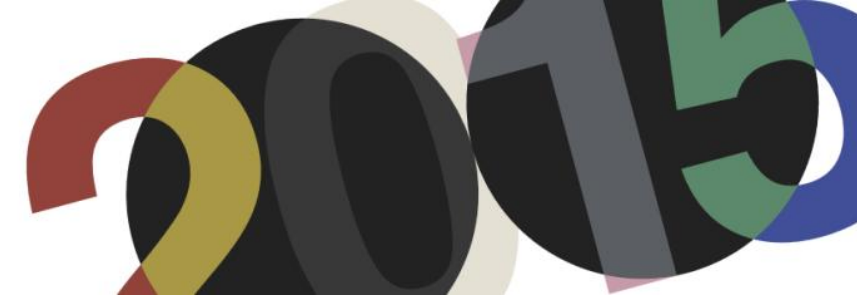

DOI: http://dx.doi.org/10.4995/LC2015.2015.787

\title{
Estrategias geométrico-matemáticas en la obra de Le Corbusier (1923-1933)
}

\author{
T. Larumbe Machín
}

Escuela Superior de Arquitectura de la Universidad de Navarra

\begin{abstract}
Resumen: En la búsqueda de un nuevo orden ideal y universal, Le Corbusier alude constantemente al empleo de los trazados reguladores como instrumentos matemáticos que controlan el impulso creador e introducen la armonía en el trabajo creado, causa de la belleza y el placer estético. La confianza puesta en los trazados como garantía del orden en arquitectura, se manifiesta en el artículo que les dedicará en la revista L'Espirit Nouveau (1921), y que, en 1923, incorpora como un capítulo más en "Vers une architecture". El objetivo de este trabajo es analizar la aplicación práctica de los trazados reguladores y otras estrategias geométricas y matemáticas en los proyectos del arquitecto suizo a lo largo de los años veinte, a partir de la aparición del famoso manifiesto. Los proyectos estudiados son los que han fijado las pautas del análisis, desvelando su evolución histórica y el alcance de su aplicación real en el transcurso de diez años.
\end{abstract}

Abstract: Along his personal search for an ideal and universal order, Le Corbusier constantly refers to the use of the tracés regulateurs, as a mathematical tool with which the architect can manage his creative impulse and as a way to introduce the harmony in architecture, what is considered the main cause of the beauty and the aesthetic pleasure. Le Corbusier reveals his faith in the tracés as guaranty of architectural order not only in the magazine L'Espirit Nouveau (1921) but also in his celebrated book "Vers une architecture" (1923). The aim of this paper is to analyze the use of the tracés regulateurs during the architect's projects throughout a decade, starting with the publication of the well know manifiesto. All the projects that had been studied have established the guidelines of the analysis, its historical evolution and the relevance of the tracès regulateurs in Le Corbusier's early works.

Palabras clave: Trazados reguladores; Geometría; Le Corbusier. Keywords: Tracés Regulateurs; Geometry; Le Corbusier.

\section{Introducción}

"El arquitecto, por el ordenamiento de las formas, obtiene un orden que es una pura creación de su espíritu; por las formas, afecta intensamente a nuestros sentidos provocando emociones plásticas, por las relaciones que crea, despierta en nosotros profundas resonancias, nos da la medida de un orden que se siente de acuerdo con el del mundo, determina reacciones diversas de nuestro espíritu y de nuestro corazón; y entonces percibimos la belleza.",

Partiendo de esta afirmación, es posible deducir dos operaciones que Le Corbusier considera fundamentales a la hora de formular un proyecto arquitectónico. En primer lugar, la selección de una serie de formas elementales "primarias o sutiles, flexibles o brutales", que constituyen la materia prima de la arquitectura. En segundo lugar, la ordenación de los distintos ingredientes plásticos con el fin de alcanzar un todo global, armónico y cohesionado, atendiendo tanto a las relaciones que las distintas figuras establecen entre sí, como a las relaciones que cada una de ellas establece con el conjunto del que forman parte; la arquitectura es un juego sabio, correcto y magnífico de volúmenes reunidos, y en este juego compositivo se encuentra el quid de su belleza. La disposición

\footnotetext{
${ }^{1}$ Le Corbusier, Hacia una arquitectura, $2^{\circ}$ Ed. Barcelona: Editorial Apóstrofe, 2006, p. XXIX

${ }^{2}$ Ibid., p. 8
} 
de los distintos elementos en el espacio debe realizarse a través de una serie de acciones sencillas: enlazar, conectar, yuxtaponer, equilibrar, mesurar, proporcionar o unificar; acciones regidas, a su vez, a través de un conjunto de principios racionales, o reglas matemáticas, que Le Corbusier eleva a la categoría de verdades absolutas.

Este código de reglas matemáticas son los trazados reguladores, que pueden definirse como un sistema de referencia sencillo que sirve para ordenar y clarificar una forma más compleja. Con el empleo de los trazados reguladores, Le Corbusier introduce un mecanismo racional de control sobre el proyecto que ya antes había prevalecido en el Renacimiento, siendo un factor determinante en las obras de Alberti y Palladio ${ }^{3}$. El interés del maestro suizo por el empleo de los trazados reguladores se manifiesta en el artículo que les dedicará en la revista L'Espirit Nouveau (1921), y que, en 1923, se vuelve a publicar en forma de capítulo en su famoso manifiesto Vers une architecture ${ }^{4}$. En él, Le Corbusier se refiere a los trazados reguladores como un medio para canalizar el impulso creador, sometiéndolo a unas reglas matemáticas; se trata de "un seguro contra la arbitrariedad" "viene a rectificar, a corregir, a depurar, a hacer consonar todas las partes sobre el mismo principio unitario". Además, la aplicación de los trazados reguladores se erige como "la operación de verificación que aprueba el trabajo creado en el entusiasmo, la prueba del nueve escolar", , es decir, los trazados reguladores se convertirán en el instrumento que justifique la belleza, la armonía y el orden del objeto creado.

Sin embargo, más allá de un estudio en torno a las implicaciones teóricas de los trazados reguladores, el objetivo de este trabajo consiste en analizar la aplicación de estrategias geométrico-matemáticas en los distintos proyectos de Le Corbusier, con la intención de extraer las estructuras de las que se sirve para introducir orden en el proyecto y, de esta manera, poder valorar su alcance en la práctica real. Para ello, se ha rastreado de manera minuciosa los proyectos elaborados por el arquitecto suizo a lo largo de una década, siguiendo un estricto orden cronológico, que toma como punto de partida el año 1923 (fecha en que se publicaba la primera edición de Vers une architecture), y como obra de referencia el archivo de planos de Le Corbusier, publicado por Garland Publishers y la Fondation Le Corbusier ${ }^{8}$ en los años 80. Como ya han señalado diversos autores, en los proyectos realizados durante esta primera etapa se fueron definiendo las bases fundamentales sobre las que el maestro suizo desarrollará el conjunto de su producción arquitectónica. Los proyectos estudiados son los que han fijado las pautas del análisis, desvelando su estructura, su propia historia y el uso que Le Corbusier hizo de los trazados reguladores en el transcurso de diez años.

\footnotetext{
${ }^{3}$ Cfr. Summerson, John, El lenguaje clásico de la arquitectura, 11 ${ }^{\text {a }}$ Ed. Barcelona: Editorial Gustavo Gili, 1998

${ }^{4}$ Cfr. Le Corbusier, Vers une arquitecture, Paris: Ediciones Crès, 1923

${ }^{5}$ Le Corbusier, Hacia una arquitectura, $2^{\circ}$ Ed. Barcelona: Editorial Apóstrofe, 2006, p. 57

${ }^{6}$ Ibid.

${ }^{7}$ Ibid.

${ }^{8}$ Le Corbusier, Le Corbusier Archive, New York: Garland Publishing, 1984
} 


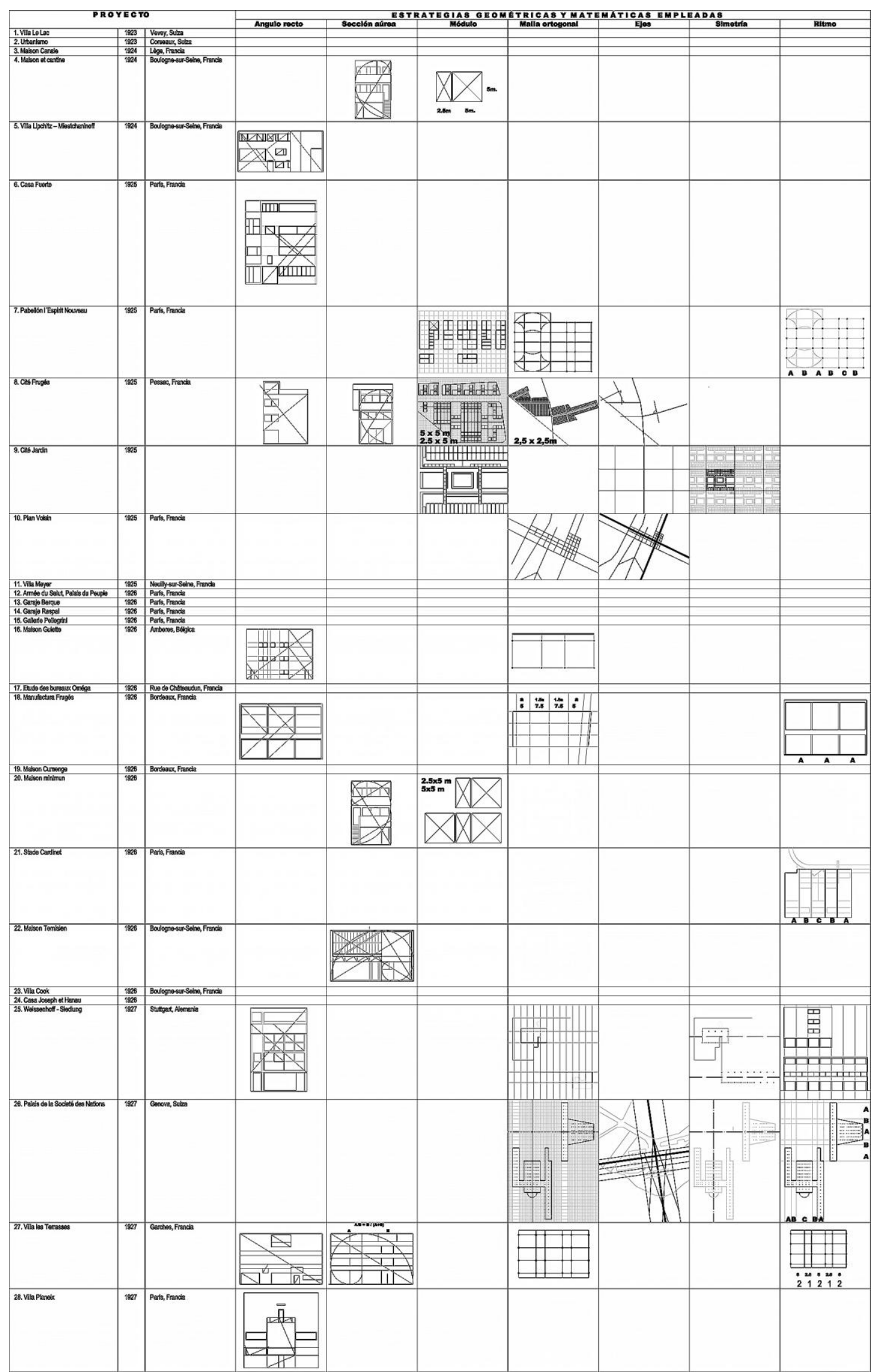

Tabla 1. Estudio de las obras de Le Corbusier realizados a partir de 1923, hasta el proyecto para la Ville Planeix, en 1927. 


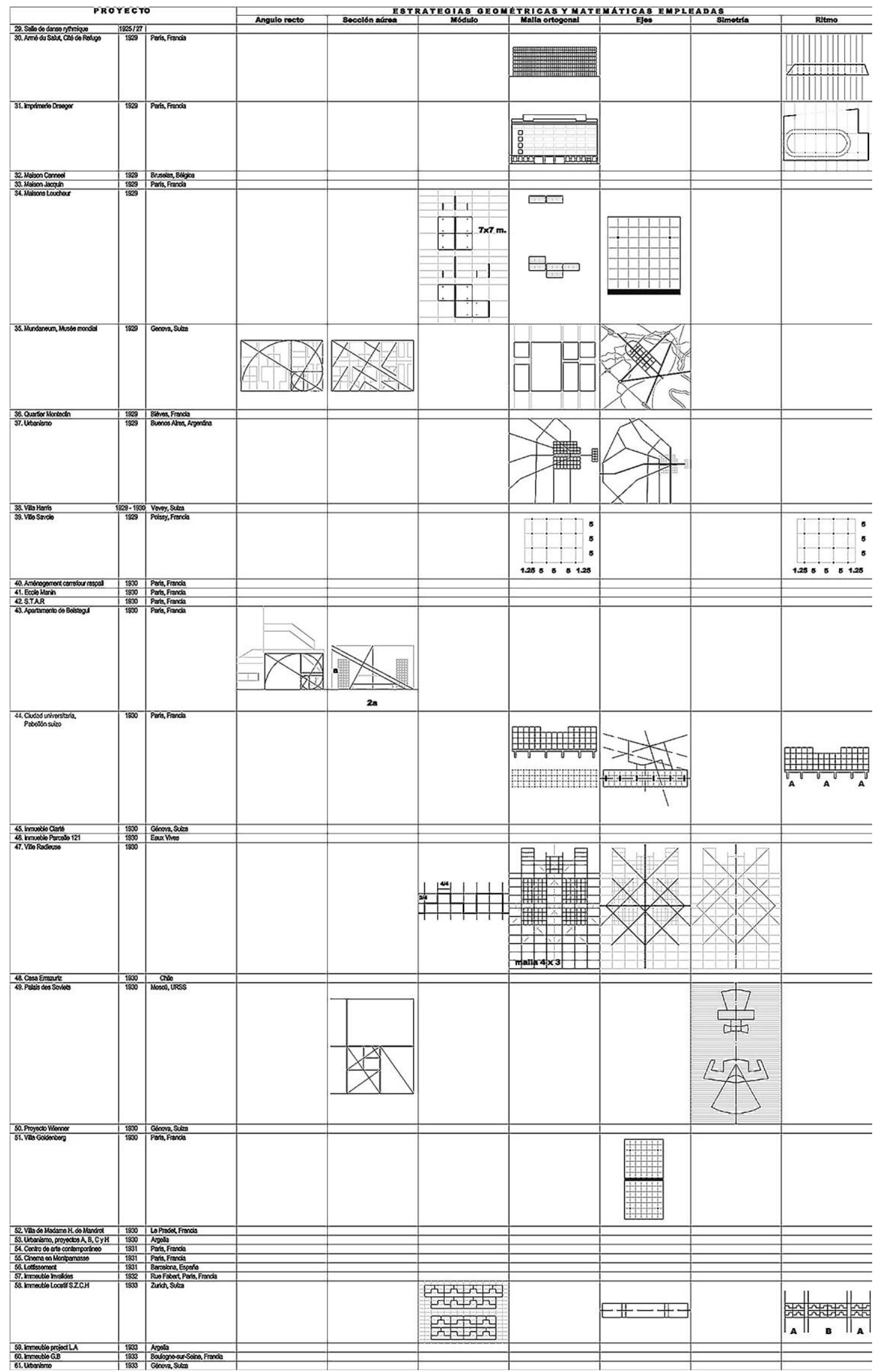

Tabla 2. Estudio de las obras de Le Corbusier, desde el proyecto para la Salle de Danse Rytmique, hasta 1933. 


\section{Estrategias geométrico-matemáticas en la obra de Le Corbusier (1923 - 1933).}

Le Corbusier emplea recursos de composición geométrico-matemáticos en 31 casos de los 61 proyectos analizados, es decir, un 50,8 \% del total. Es posible distinguir seis tipos de estrategias diferentes, que son referidas por Le Corbusier en sus textos teóricos: el ángulo recto (se emplea en 17 casos, un 54.8\% de los 31 proyectos en los que se ha observado la impronta de los trazados reguladores); la proporción aurea (8 proyectos, $25.8 \%$ ); el módulo (8 proyectos, $25.8 \%$ ); la malla ortogonal (17 proyectos, $54.8 \%$ ); los ejes (12 proyectos, $38,7 \%$ ); el ritmo (12 proyectos, $38,7 \%$ ); y las relaciones de simetría bilateral (4 proyectos, un $12.9 \%$ del total). A su vez, es posible agrupar las distintas estrategias empleadas en dos categorías diferentes, atendiendo a su naturaleza matemática; estrategias aritméticas y reglas geométricas (aunque cabe señalar que la frontera entre ellas, en algunas ocasiones, es difusa). Por un lado, el ritmo vertical que compone las fachadas o la proporción numérica existente entre los dos ejes de las retículas ortogonales son reglas compositivas basadas en las relaciones aritméticas entre sus componentes. Por otro lado, el ángulo recto, el número aureo, la disposición de los ejes y las relaciones de simetría son trazados de naturaleza geométrica. Del módulo se derivan una serie de relaciones tanto numéricas (cuando el módulo es una unidad métrica -como, por ejemplo, las distancias entre pilotis-), como geométricas (cuando la unidad básica de referencia es una figura geométrica, por ejemplo, los módulos cuadrados empleados en las viviendas seriadas).

Además, es posible realizar una nueva clasificación de las distintas estrategias compositivas atendiendo a la escala del proyecto sobre el cual se aplican dichas reglas: desde el diseño de pequeños objetos, hasta la elaboración de planes urbanos, pasando por el diseño de edificios singulares. En el diseño de elementos de pequeña escala (esto es, mobiliario y detalles constructivos), Le Corbusier recurre al empleo del ángulo recto y el sistema modular. En los proyectos de planeamiento urbano, podemos observar el uso de retículas ortogonales, ejes y módulos. Finalmente, dentro de los proyectos de edificación realizados por el maestro suizo, es posible distinguir dos ámbitos distintos donde se aplican las reglas geométrico-matemáticas, que se corresponden con dos de las tres advertencias citadas por Le Corbusier'; la composición de la superficie o el alzado y la ordenación de la planta o el plan generador. En todos los ejemplos analizados, los trazados reguladores se emplean como herramienta de control sobre superficies planas, en ningún caso sirve para moldear el volumen.

\section{Descripción de las distintas estrategias geométrico-matemáticas empleadas.}

\section{1. Ángulo Recto.}

Diversos autores se han referido con frecuencia a Le Corbusier como autor del Poema del ángulo recto, pero en numerosas ocasiones esta imagen es restrictiva y limitada, ya que se asocia exclusivamente con su gusto por la pureza formal, o con el empleo de volúmenes prismáticos y retículas ortogonales en sus obras. Sin embargo, en sus textos, Le Corbusier alude frecuentemente al uso del ángulo recto como elemento que sirve para definir un sistema de proporciones ( “... iHe ahí la proporción!/la proporción que pone /orden en nuestras / relaciones con / lo circundante ${ }^{\jmath 10}$ ), y como herramienta de composición que sitúa los objetos dentro del espacio del plano ${ }^{11}$. La aplicación del ángulo recto en los proyectos que se han analizado viene a corroborar estas afirmaciones. Le Corbusier hará uso del ángulo recto a la hora de componer, no las plantas, sino los alzados de la mayor parte de los proyectos realizados en su primera etapa, así como los alzados de todas sus célebres viviendas: el proyecto

\footnotetext{
${ }^{9}$ Cfr. Le Corbusier, Hacia una arquitectura, $2^{\circ}$ Ed. Barcelona: Editorial Apóstrofe, 2006, p. 31

${ }^{10}$ Le Corbusier, Poema del ángulo recto, Madrid: Círculo de Bellas Artes, 2006

${ }^{11}$ Cfr. Le Corbusier, Hacia una arquitectura, $2^{\circ}$ Ed. Barcelona: Editorial Apóstrofe, 2006, p. 59
} 
para la Ville Lipchitz-Miestchaninoff (1924); la casa Fuerte, en París (1925); las viviendas de la Cité Frugés, en Pessac (1925); la Maison Guiette, en Amberes (1926); la manufactura Frugés (1926); los edificios de la Wiessenhoff, en Stuttgart (1927); la Ville en Garches (1927); la Ville Planeix, en Burdeos (1927); y el apartamento Beistegui, en París (1930).

El lugar del ángulo recto es un punto presente en el plano de la fachada que queda definido por dos diagonales que se cruzan entre sí, formando un ángulo de $90^{\circ}$. Por este punto pasará una línea virtual (tanto horizontal como vertical), que servirá de referencia para situar los distintos objetos contenidos dentro de la superficie de los paramentos verticales. Al mismo tiempo, el juego de diagonales ortogonales define la relación proporcional entre los lados de la fachada, y su uso se reitera con el objetivo de dimensionar todos los huecos que aparecen en el alzado, obteniendo como resultado una composición a base de rectángulos semejantes entre sí. El juego de proporciones generadas a partir del ángulo recto se aplicará sobre las superficies verticales (planos de fachada, paramentos interiores y despiece de ventanas), y podemos relacionar su empleo con el principio formal de la fachada libre, uno de los cinco puntos enunciados por el maestro suizo para la nueva arquitectura. Tras haber alcanzado una absoluta autonomía en las fachadas, siendo éstas independientes de cualquier condicionante estructural, y libres también de cualquier imperativo constructivo que pudiera encorsetar la composición del alzado, le Corbusier recurrirá al ángulo recto y se servirá de él para establecer unas reglas de juego con las que dibujar sus composiciones sobre el "papel en blanco".
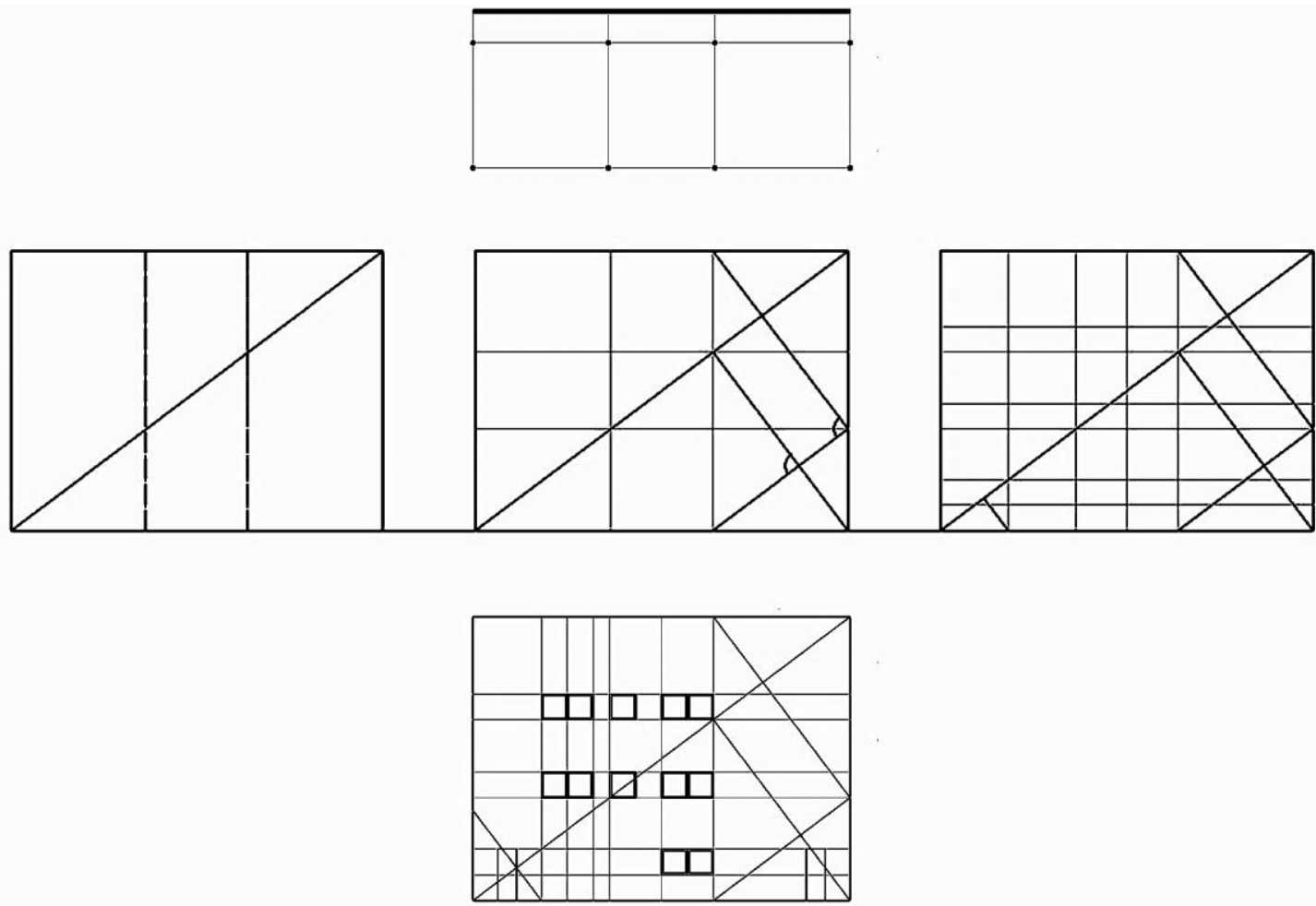

1. Maison Guiette. Amberes, 1926. El trazado regulador del proyecto, de 6 metros de crujía, se desarrolla a partir de su esquema estructural. El ángulo recto, este caso, generará una serie de líneas virtuales que guíen el dibujo del alzado. La composición de los alzados vendrán determinados por los ejes de los pilares, ocultos tras el paramento de fachada, que dibujan unas líneas auxiliares verticales sobre el "papel en blanco". La aplicación del ángulo recto se empleará, a su vez, para determinar la posición exacta donde deban situarse las directrices horizontales, que vendrán a sumarse a las guías verticales para formar un damero virtual en el que se irán situando los huecos de la vivienda. El ángulo recto, por otra parte, no sólo regula la proporción del plano de fachada, sino que también dicta la proporción a la que han de ajustarse cada uno de sus huecos. 


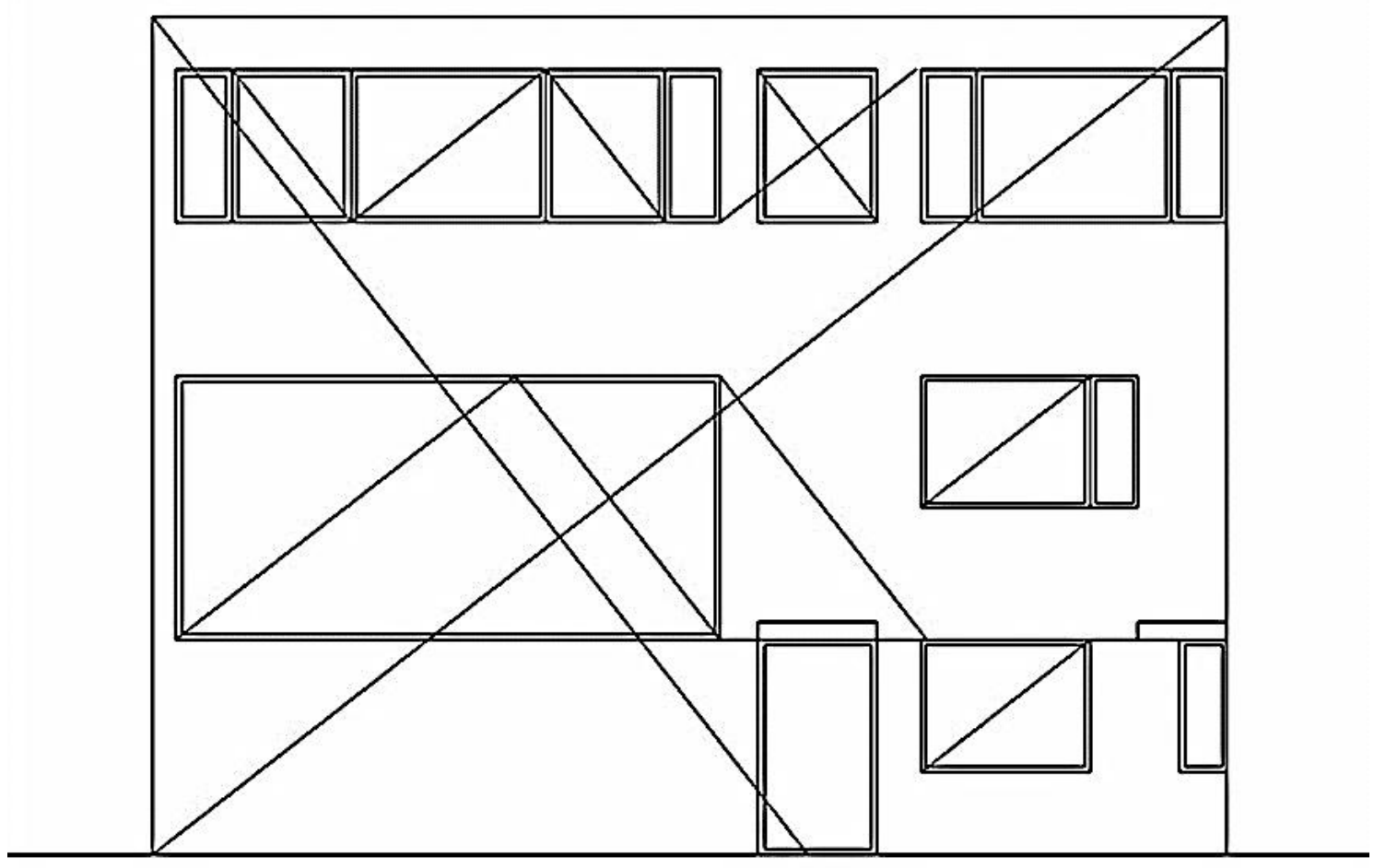

2. Maison Lipchizt Miestchaninoff. París, 1925. La complejidad del solar en el que se sitúa la intervención (un polígono irregular) impondrá su propio orden a la planta de la vivienda y a las dimensiones del volumen. Sin embargo, libre de restricciones estructurales que puedan condicionar el diseño de los alzados, Le Corbusier recurre al ángulo recto para establecer un sistema objetivo de proporciones semejantes entre el paño de fachada y los huecos que en él se sitúan, a través de juegos diagonales, paralelas y perpendiculares. Los alzados son elaborados como si de composiciones pictóricas puristas se tratase.

\section{La sección aurea.}

Dentro del empleo del ángulo recto como trazado regulador y de las relaciones proporcionales que derivan de su uso, hay un caso especialmente significativo: el rectángulo aureo, esto es, aquel en el que la razón proporcional entre sus lados es el número dorado. A pesar de la propaganda que el autor hace de la proporción aurea en la obra El Modulor ${ }^{12}$, sólo encontramos esta proporción en un 25,8\% de los proyectos realizados entre 1923 y 1933, en los que aparece la impronta de los trazados reguladores. Al igual que sucedía con los trazados basados en el empleo del ángulo recto, Le Corbusier recurre a la sección aurea para establecer relaciones proporcionales entre los huecos que componen las fachadas de los distintos proyectos, y para relacionar cada uno de estos elementos con el todo del que forman parte. Este recurso, además de justificar la belleza de la solución empleada, será la respuesta científica con la que trazar unas líneas virtuales significativas en el plano en blanco que sirvan para regular el diseño del alzado. Por tanto, el diseño de los alzados y el modelado de los volúmenes se erige como el ámbito de aplicación por excelencia de la proporción aurea. Encontramos huellas de su uso en el proyecto para la Maison Cantine, en Boulogne-sur-Seine (1924) que, años más tarde, servirá de modelo de referencia para los estándares de vivienda mínima; las viviendas de Pessac (1925) y la Maison Minimun (1926). Además, la proporción aurea servirá para modelar los volúmenes que componen la Maison Ternisien, en

\footnotetext{
${ }^{12}$ Cfr. Le Corbusier, El Modulor, Barcelona: Editorial Poseidón, 1976
} 
Boulogne-sur-Seine (1926) y también para diseñar el despiece de las carpinterías del apartamento Beistegui, al que aludíamos en el apartado anterior, y los bastidores que construyen los huecos del proyecto para el Palacio de los Soviets (1930).

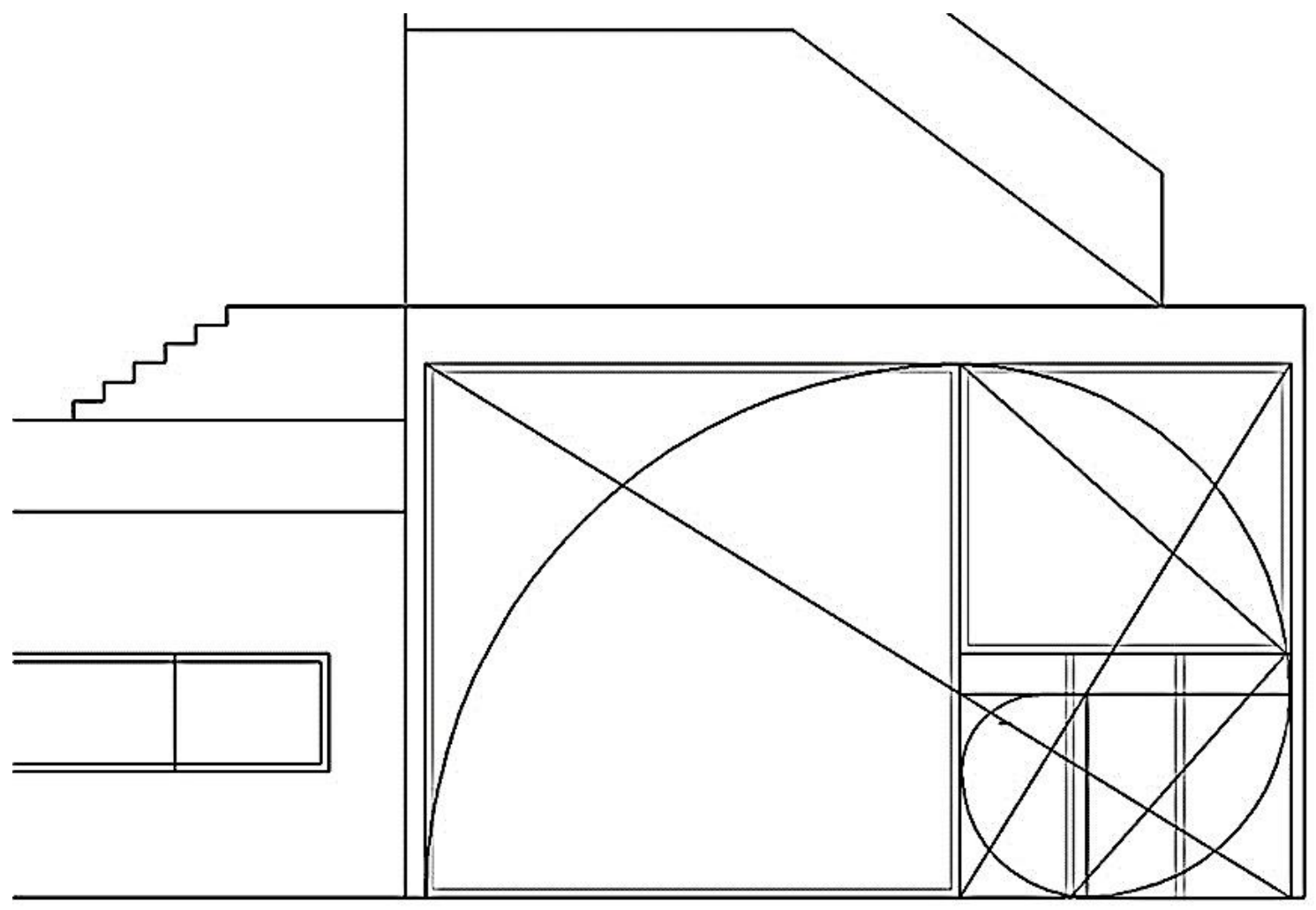

3. Apartamento de Beistegui. París, 1930. Le Corbusier hará uso del ángulo recto y la proporción aurea en el diseño de los distintos detalle constructivos, como el despiece de las carpinterías del gran ventanal que vierte a la terraza del ático, o la composición de los paramentos interiores de la casa.

En los proyectos de la célebre Ville les Terrasses y el inmueble Les Invalides, el número de oro no sólo servirá para proporcionar y componer los alzados longitudinales del volumen, sino también para configurar su estructura, condicionando la distancia entre pilares y estableciendo, con ello, un ritmo basado en la serie Fibonacci. Además, en la Ville Les Terrasses, la composición de los pilotis guardará una relación rítmica simétrica, ABABA, legado de la composición académica. Para acentuar el carácter significativo de la obra, Le Corbusier recurrirá a las medidas de 5 metros, $5 / 2$ y 5/4, empleados también en proyectos anteriores, en su búsqueda de dimensiones ideales. La aplicación de la razón aurea se limitará generalmente a regir la composición de las superficies verticales en los distintos proyectos, ya sean fachadas, particiones interiores, o despieces de los ventanales. De manera significativa, la sección aurea también aparece como trazado para ordenar la planta en dos proyectos particulares; el proyecto para el Museo Mundial y el centro de arte contemporáneo de París (en ambos casos, le Corbusier emplea una tipología en espiral para el diseño del edificio destinado a museo, que se convertirá en un arquetipo personal). En todos estos proyectos, la proporción armónica dotará al proyecto de un fuerte contenido simbólico. 


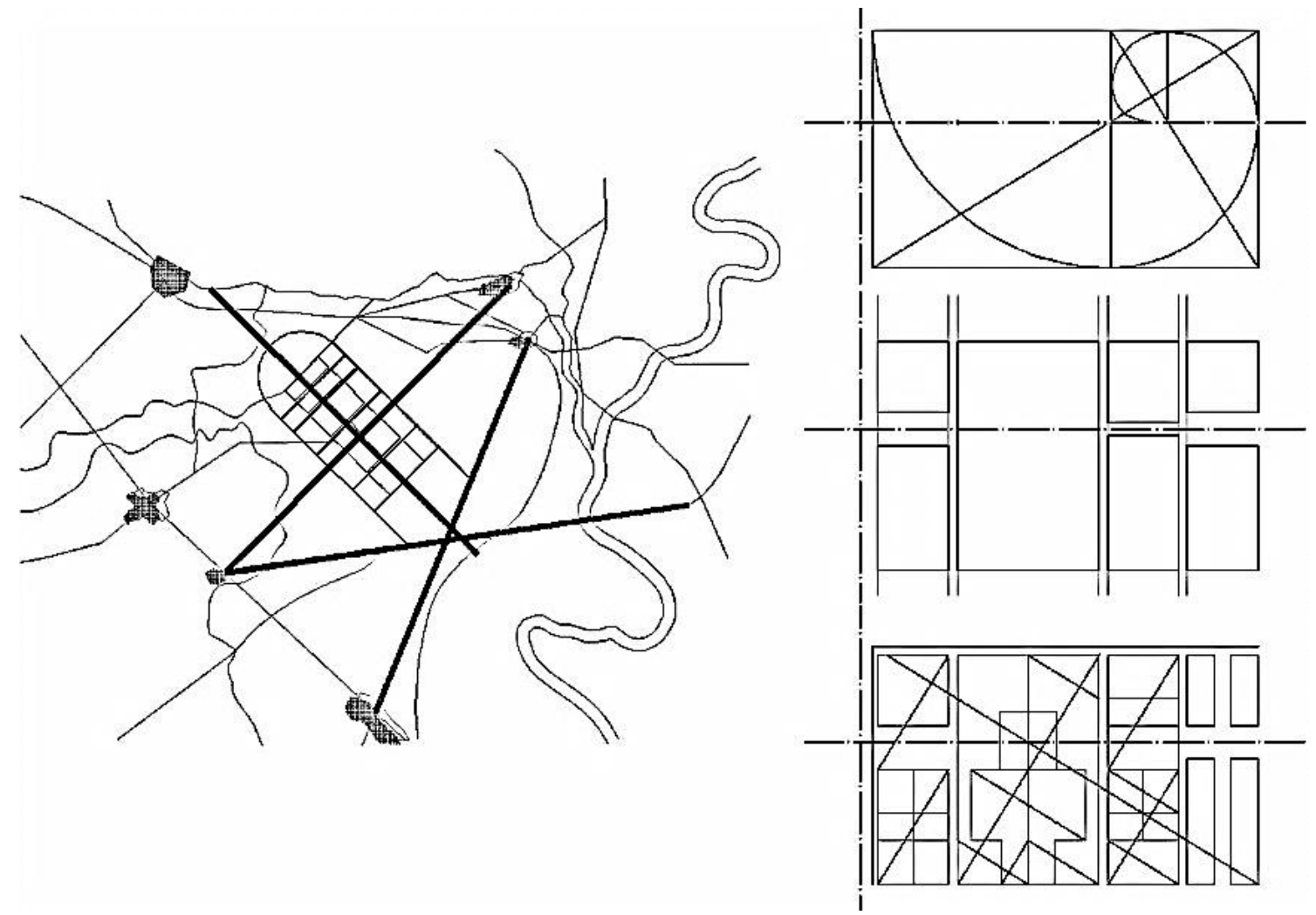

4. Mundaneum. 1929. En este proyecto, Le Corbusier recurre a un sistema de ejes para situar el plan del nuevo complejo cultural en relación al contexto en el que se inserta y para determinar el eje axial del que se servirá para conectar todos los volúmenes del conjunto entre sí. Se trata de uno de los pocos proyectos en el que Le Corbusier aplica un trazado regulador geométrico basado en la proporción aurea y el ángulo recto para definir el plan. Este hecho demuestra la importancia significativa que el arquitecto atribuye al proyecto. La pieza principal del museo se desarrolla a partir de un esquema en espiral, cuyo crecimiento se basa en la serie de Fibonacci.

\section{El Módulo.}

"Un módulo mide y unifica; un trazado regulador construye y satisface." El módulo es una figura empleada como unidad de referencia que sirve para establecer relaciones métricas a partir de combinaciones numéricas sencillas; múltiplos y derivados. Pero al mismo tiempo, Le Corbusier lo emplea como un trazado regulador, puesto que de su propia naturaleza derivan una serie de reglas de composición que confieren un orden unitario al conjunto. Las combinaciones modulares aparecen asociadas desde un primer momento a los sistemas de construcción estandarizados, como se puede observar en su diseño de muebles para el pabellón de l'Espirit Nouveau (1925). Se tratan tanto de unidades volumétricas, como de unidades superficiales. Le Corbusier recurre con frecuencia a la célula-tipo cúbica de $5 \times 5 \times 5$ metros, que aplicará en los proyectos para sus viviendas experimentales; la Maison et Cantine, la Maison Minimun, las viviendas de la Cité Frugés, o la Maison Loucheur, y las distintas combinaciones que del módulo cuadrado de la planta que de ella derivan; $1 / 2$ ( 5 x 2.5 m.), 1/4, ( 5 x 1,25 m.), 3/2 ( $5 \times 7.5$ metros), etc. Pero el módulo también puede tener naturaleza lineal, como unidad que sirve para dividir un eje y generar un desarrollo rítmico. Le Corbusier confiará de nuevo en el número 5 como unidad lineal con la que medir y unificar su proyecto piloto de viviendas colectivas (la Weissenhoff de Stuttgart), que se corresponde con la distancia que guardan sus pilares entre sí. Por último, en los croquis iniciales de su célebre villa, la Ville Savoie de París (1929), de nuevo aparece la retícula ideal de 5 x 5 metros como base sobre la que se distribuye la planta. 
$5 \mathrm{~m}$.
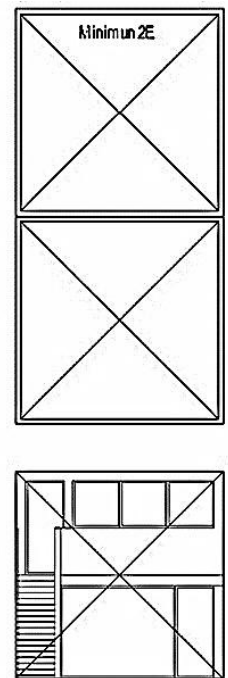

$2,5 \mathrm{~m}$.
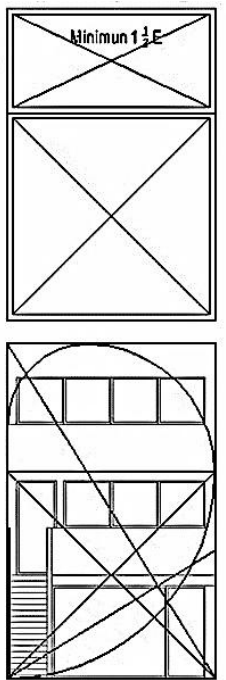
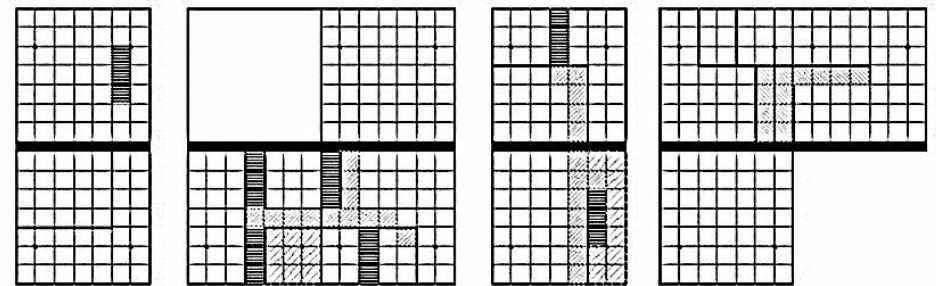

$+H+H$

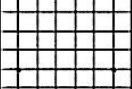

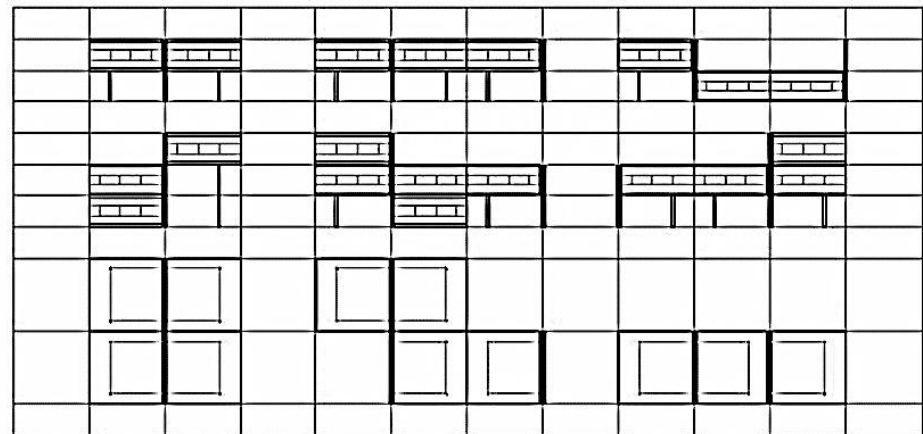

5. Maison Minimum, 1926 (arriba) y Maison Loucheur, 1929 (abajo). En el proyecto para la Maison Minimum, Le Corbusier recurre al módulo estándar que había empleado en numerosas ocasiones anteriormente (entre otras, el proyecto para la Maison Cantine, en Boulogne-sur-Seine, de 1924, y en la Cité Frugés, en Pessac, en 1925); el cubo de 5 x 5 x 5 metros. De esta célula básica se deducen una serie de combinaciones en planta (mitad del cuadrado, doble cuadrado, tres cuadrados, cuadrado y medio...), generando con ello nuevos modelos estándar de vivienda mínima. Por otra parte, los distintos alzados posibles parten también de la unidad 5 x 5 , y sus variaciones geométricas; el cuadrado y su rectángulo aureo. Del uso del módulo cuadrado se derivan unas reglas geométricas y aritméticas muy sencillas con las que se pueden aplicar en la configuración de barrios enteros (Pessac), de modo que esa unidad mínima se convierte, en sí misma, en un elemento geométrico regulador. La misma estrategia encontramos en los experimentos derivados de la Maison Loucheur. En este caso, la unidad básica de medida consistirá en un cuadrado de 7 x 7 metros, dividida, a su vez, en una cuadrícula, para facilitar su distribución. 


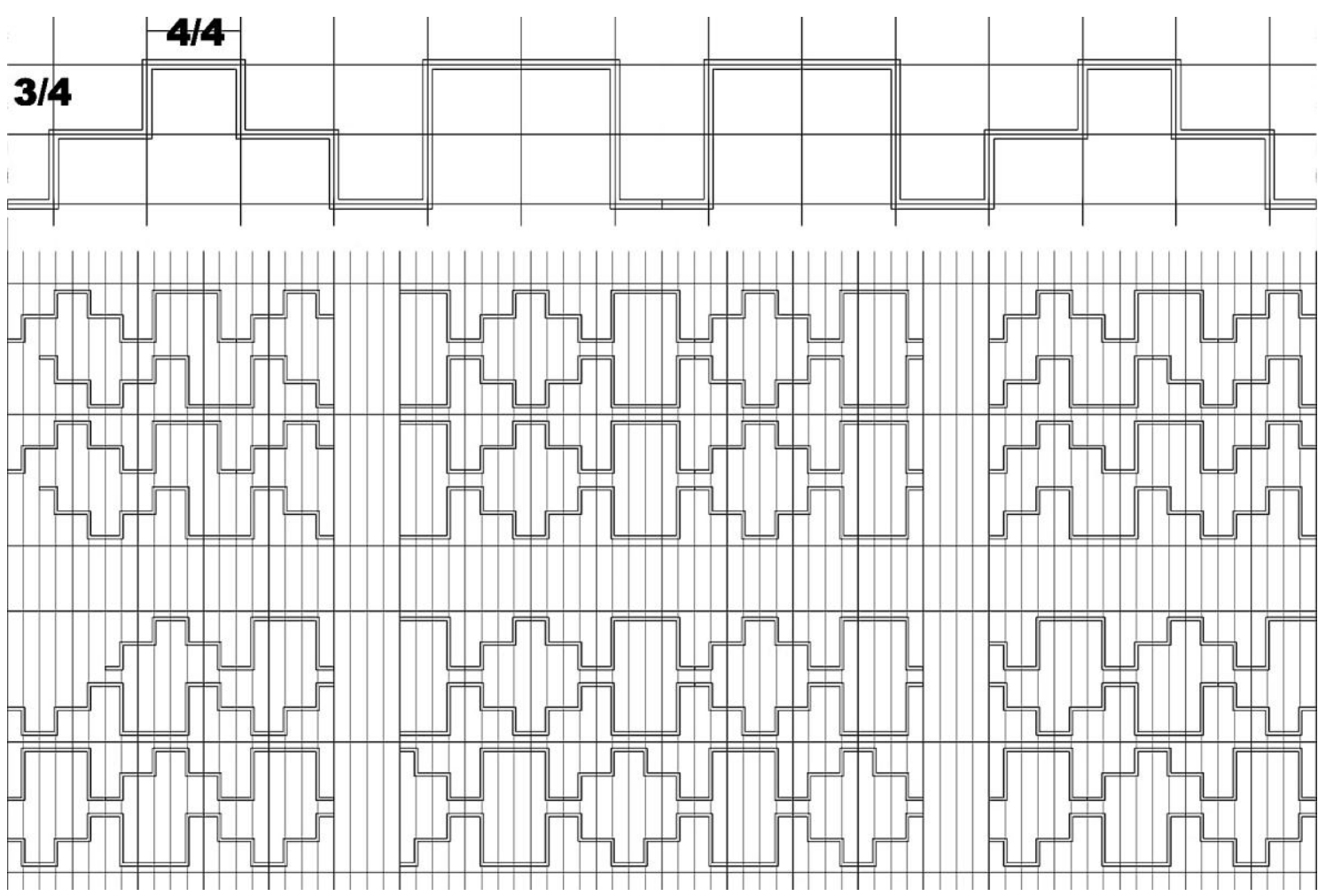

6. Ville Radieuse, 1930 (detalle). Detalle de las posibles supermanzanas que podrían generarse a partir de la combinación de un modelo de bloque residencial dispuesto en redant.

\section{Las mallas ortogonales.}

Mientras que en gran parte de sus textos Le Corbusier cita continuamente diversos trazados reguladores como el lugar del ángulo recto, el eje o los juegos proporciones basados en la razón aurea, no aparecen alusiones explícitas al uso de las mallas ortogonales como esquema compositivo. En su manifiesto hacia una nueva arquitectura, el maestro suizo sólo se referirá al empleo de la malla de manera indirecta al hablar del orden ortogonal que necesariamente ha de regular los nuevos barrios de viviendas en serie ${ }^{13}$, y también mencionará la belleza de la geometría cuadriculada presente en las fachadas de las construcciones fabriles norteamericanas, que deriva de la estricta aplicación de los principios funcionales y constructivos:

"Problema de la época y de la estética contemporánea: todo conduce a la reinstauración de los volúmenes simples (...) La superficie, agujereada por las necesidades del destino, debe tomar las generatrices acusatrices de esas formas simples. Esas acusatrices son prácticamente el tablero de damas o la cuadrícula: Fábricas norteamericanas. ¡Pero esta geometría de miedo! Sin seguir una idea arquitectónica, sino simplemente guiados por las necesidades de un programa imperativo, los ingenieros de nuestros días recurren a las generatrices acusatrices de los volúmenes: muestran el camino y crean realidades plásticas, claras y límpidas, brindando paz a los ojos y los goces de la geometría al espíritu"14.

Sin duda, a finales de los años treinta, Le Corbusier seguirá el ejemplo de las construcciones fabriles norteamericanas en las fachadas sus numerosos proyectos de inspiración industrial, como, por ejemplo, la manufactura Frugés de Burdeos (1926); el edificio para la Armé du Salut, en la Cité du Refuge de París (1929);

\footnotetext{
${ }^{13}$ Cfr. Le Corbusier, Hacia una arquitectura, $2^{\circ}$ Ed. Barcelona: Editorial Apóstrofe, 2006, p. 193

${ }^{14}$ Le Corbusier, Hacia una arquitectura, $2^{\circ}$ Ed. Barcelona: Editorial Apóstrofe, 2006, p. 28
} 
la Imprimerie Draeger, también en París (1929); o el pabellón suizo de la Ciudad Universitaria (1930). Sin embargo, años atrás, ya se había servido de las de las mallas ortogonales para regular la composición y la distribución de las plantas en sus grandes proyectos iniciales, como, por ejemplo, el pabellón de l'Espirit Nouveau (1925); el proyecto para el palacio de la Sociedad de Naciones, en Génova (1927); la villa Les Terrasses en Garches (1927); o la Ville Savoie (1929). El uso de las mallas ortogonales como estrategia geométrica de diseño, siempre estará relacionada con el empleo de una estructura porticada a base de pilares. También encontramos una fuerte presencia de la malla ortogonal en la estructura subyacente de sus proyectos urbanos, tanto reales como utópicos, como el complejo residencial de Pessac; el proyecto de Ciudad Jardín (1925); el Plan Voisin (1925); o la Ville Radieuse (1930).
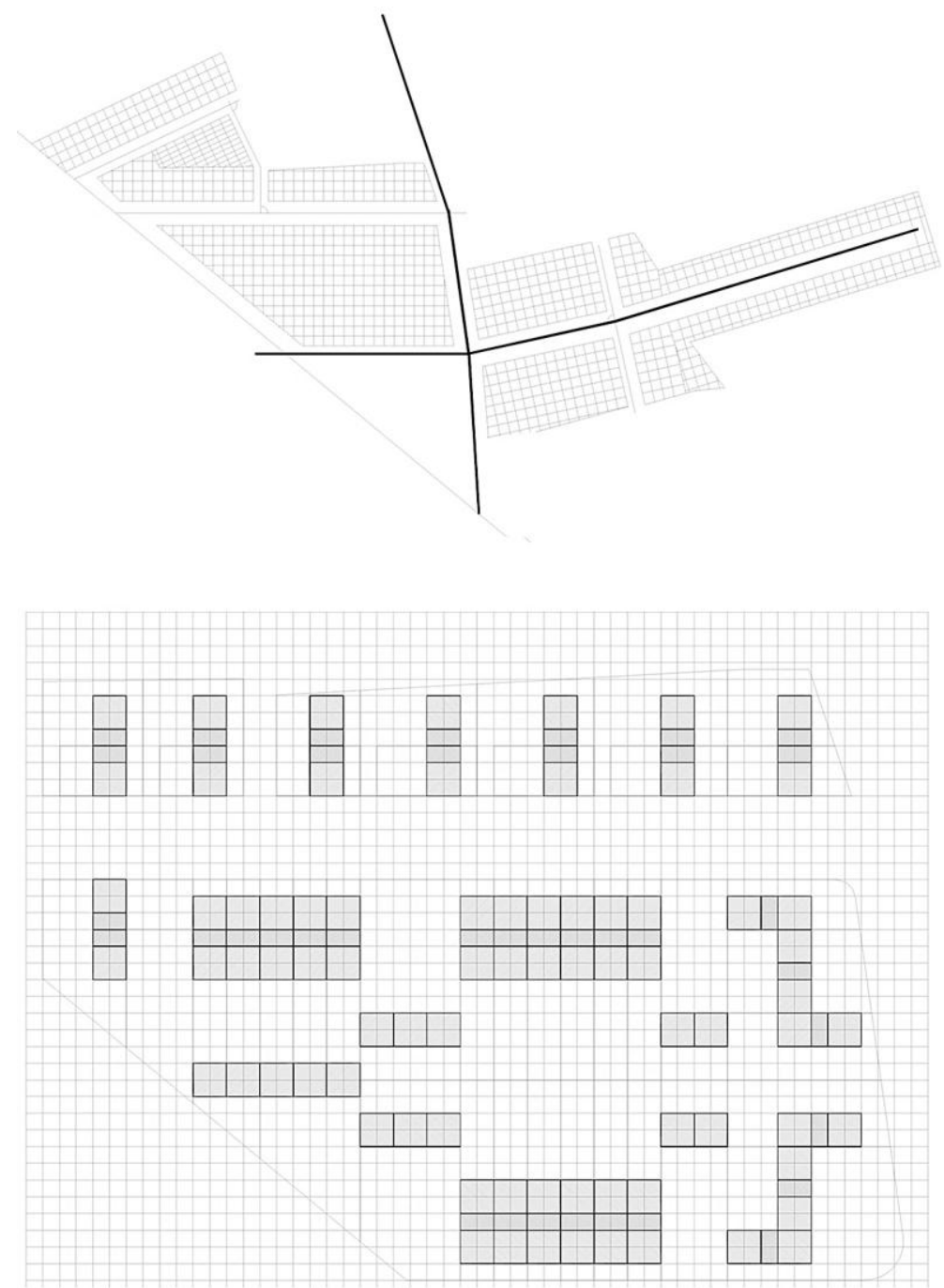

7. Cité Frugés, Pessac, 1926. Complejo urbano (arriba) y detalle de manzana (abajo). En Pessac, unos ejes trazan las directrices generales del proyecto, poniendo en relación la intervención con el resto del territorio y establecen, a su vez, una jerarquía en las calles. Le Corbusier empleará su modelo estándar para la vivienda mínima, que colocará sobre una malla ortogonal de 5x5 metros, que sirve para regular y unificar el conjunto, sobre la que dibujará las divisiones catastrales. 

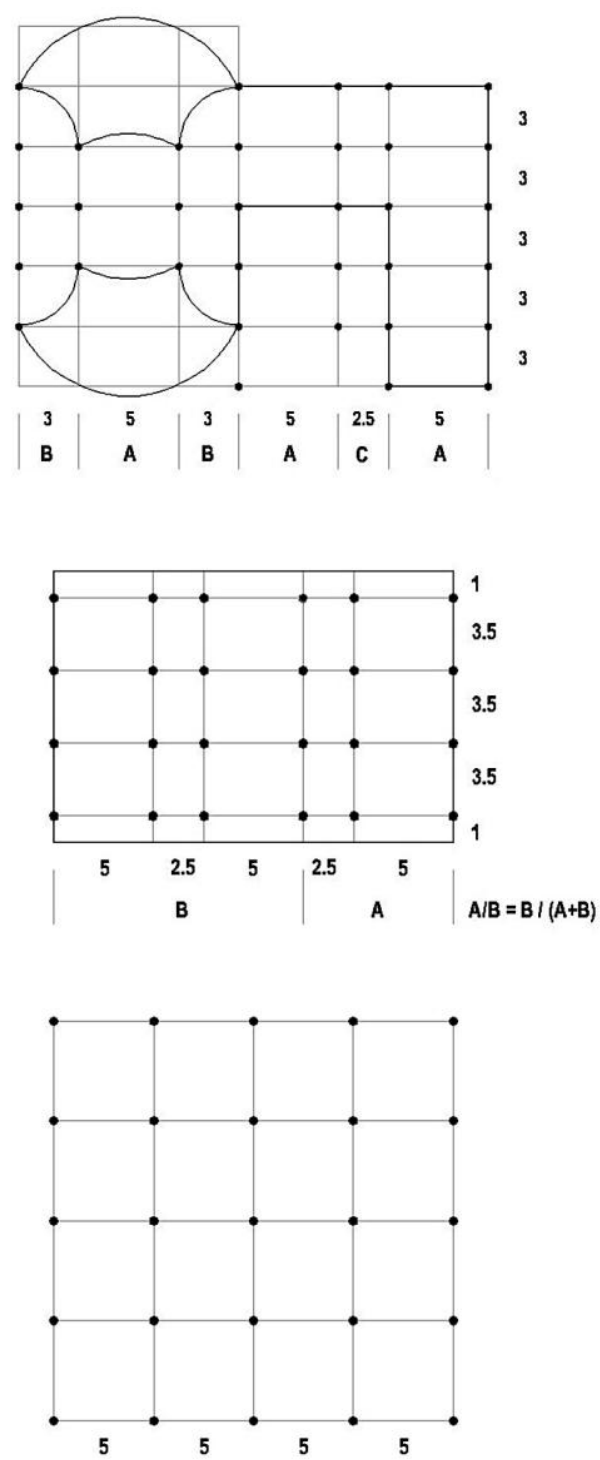

8. Retículas estructurales correspondientes al Pabellón L'Espirit Nouveau, París, 1925 (Arriba); Ville Les Terrasses, Garches, 1927 (en medio); y Ville Savoie, París, 1929 (debajo). En los tres casos, el sistema estructural empleado, a base de pilotis, es un sistema normalizado que contiene en sí mismo un orden, sobre el que se producirá el ritmo y la variación. En los tres proyectos, se revela el interés de Le Corbusier por el número 5, en su objetivo de imprimir dimensiones ideales en sus proyectos.

\section{Ejes, ritmo y simetrías.}

Los ejes son un recurso compositivo lineal que encontramos en un 25,8\% de los proyectos analizados, está directamente relacionado con otro tipo de instrumentos de medida y ordenación, como el ritmo o la simetría, y su empleo pone en evidencia el legado del clasicismo académico francés en la obra de Le Corbusier. Los ejes son utilizados por el arquitecto suizo como trazado que regula tanto las plantas de los edificios, como los planes urbanos de gran escala. Aparecen en los diversos croquis de proyecto desde el momento de la concepción de las obras y en ellos se expresan intenciones distintas en función de las necesidades a resolver, tal y como el autor explica en sus textos: "El eje es el que pone orden en la arquitectura. Poner orden, es comenzar una obra (...) 
En arquitectura, es preciso que un eje tenga un objetivo". ${ }^{15}$ Los proyectos analizados vienen a confirmar esta afirmación, y en ellos se distinguen tres usos distintos del eje: en primer lugar, los ejes sirven para relacionar la intervención arquitectónica con el territorio en el que se inserta; en segundo lugar, se emplean para conectar distintas partes del programa en aquellos proyectos de gran envergadura y complejidad, estableciendo una relación jerárquica entre ellas; por último, el eje aparece como instrumento de medida reglado, a lo largo del cual se desarrollará un esquema rítmico.

El eje, como instrumento que conecta el nuevo tejido urbano con su contexto, está presente en todos los proyectos de planificación urbana elaborados por el maestro suizo. Su empleo es fundamental en aquellos proyectos adscritos a un contexto determinado, como el Plan Voisin, el plan para Buenos Aires o proyecto para el Mundaneum, y no tanto en otros esquemas urbanos ideales y desarraigados, como la Ciudad Jardín (1925) o la Ville Radieuse (1930), en los que el eje es utilizado como un elemento puramente compositivo. Para Le Corbusier, "las ciudades deberán ser concebidas y trazadas en toda su extensión, como fueron trazados los templos de Oriente y como fueron ordenados los Inválidos o el Versalles de Luis XIV"16. Con este objetivo, el arquitecto empleará los mismos principios geométricos que regulan los modelos urbanos barrocos, cuyos trazados, en forma de tridente, se extienden a lo largo de todo el territorio, colonizando el lugar e instaurando en él un nuevo orden. También se servirá de los ejes ortogonales, cardus y decumanus, empleados como referencia en el trazado de las ciudades clásicas.
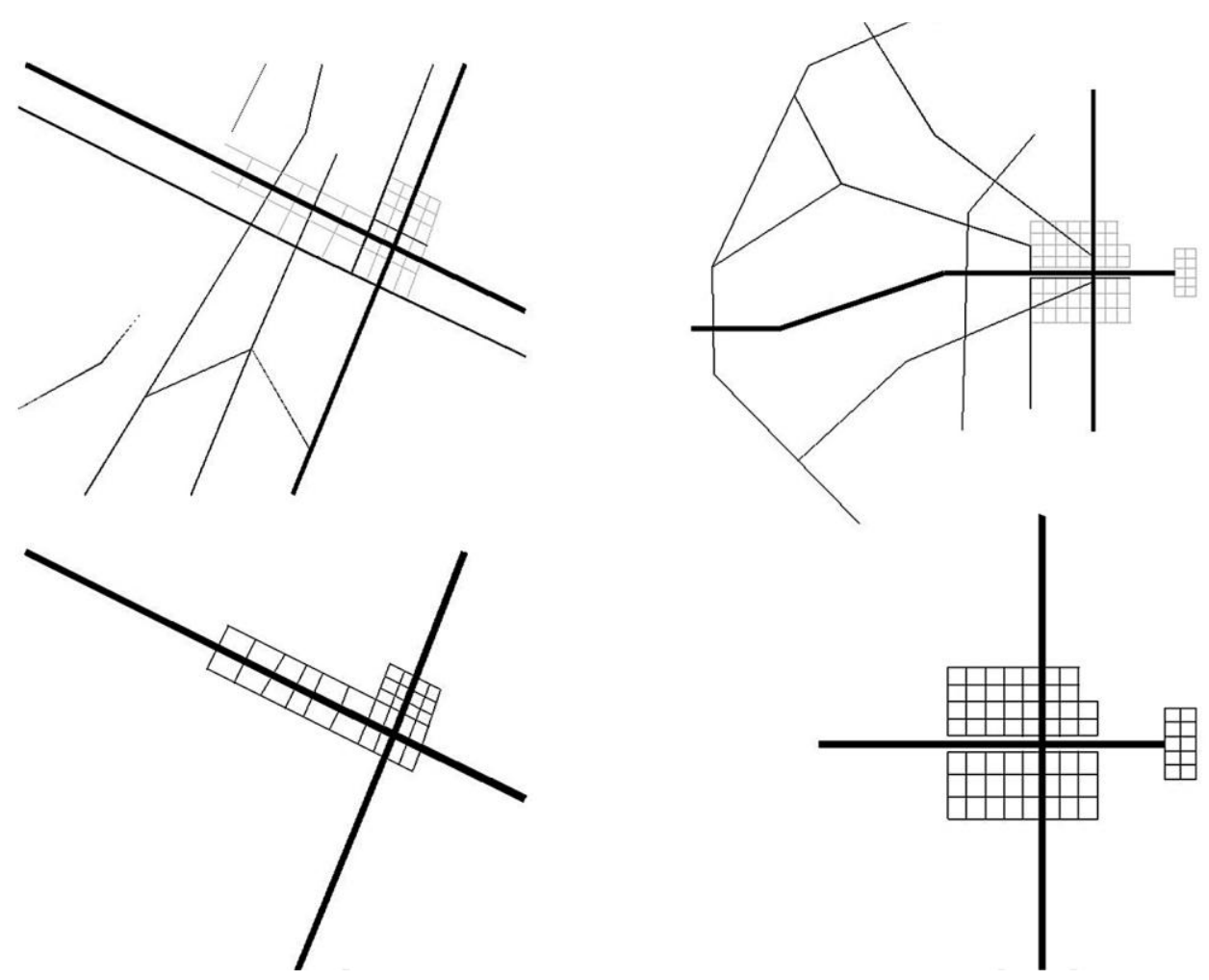

9. Plan Voisin, París, 1925 (izquierda); Plan para Buenos Aires, Argentina, 1929 (derecha). En los planes urbanos aparece el eje como trazado que sirve para relacionar el nuevo entramado con el territorio en el que se inserta. En estos proyectos Le Corbusier recurre al empleo de un sistema de ejes barrocos en forma de tridente, y unos ejes ortogonales (vestigios del esquema romano formado por las vías cardus y decumanos) que se convierten en las directrices principales del nuevo plan y determinan la dirección del entramado urbano, que adoptará la forma de damero.

\footnotetext{
${ }^{15}$ Le Corbusier, Hacia una arquitectura, $2^{\circ}$ Ed. Barcelona: Editorial Apóstrofe, 2006, p. 151

${ }^{16}$ Ibid.
} 
En segundo lugar, Le Corbusier recurre al esquema axial en aquellos proyectos de gran envergadura y complejidad programática con la intención de ordenar los volúmenes que componen el conjunto; "el eje no es aquí una aridez teórica, sino que une los volúmenes capitales y netamente escritos y diferenciados los unos de los otros" "17. Su empleo se manifiesta en los grandes complejos edificatorios como el Palais de la Societé des Nations, en Génova (1927); la Siedlung de Stuttgart (1927); el pabellón Suizo de la Ciudad Universitaria (1930); el proyecto para el Palacio de los Soviets de Moscú (1930). En este sentido, el eje es también empleado para referir un programa muy complejo a unas relaciones muy sencillas entre sus partes, estableciendo una jerarquía, como se observa en el proyecto para el Palacio de las Naciones, donde el eje, además, determina una línea virtual de simetría que imprime al proyecto un carácter monumental; "La ordenación es la jerarquía de los ejes, por lo tanto, la jerarquía de los fines, la clasificación de las intenciones" ${ }^{\prime 1}$. En estos casos, el eje se convierte en el elemento generador en el que se expresan las intenciones del proyecto.

Por último, Le Corbusier recurre al eje como un instrumento de medición, dividido en una serie de unidades de longitud, que pueden ser agrupadas en conjuntos armónicos para introducir, de este modo, un ritmo en la composición del alzado. Por tanto, el ritmo también se convierte un esquema regulador, un conjunto de reglas que rigen las relaciones entre los distintos elementos de composición arquitectónica, en este caso, a lo largo de un eje lineal. El autor defiende que el ritmo "es un estado de equilibrio que procede de simetrías simples o complejas o de compensaciones sabias. El ritmo es una ecuación",19, y, como toda ecuación, exige repartir cantidades iguales entre sus miembros. Por tanto, Le Corbusier recurre en numerosos casos a la simetría bilateral, especialmente en sus primeras obras, repitiendo esquemas como: ABA, en la Ville Planeix de Burdeos (1927), el Pabellón Suizo de la Ciudad Universitaria (1930) o el Inmeuble Locatif, de Zurich (1933); AAA, en la fachada de la manufactura Frugés, (1926); ABCBA, en el estadio Cadinet, de París (1926); ABABA en el Palacio de la Sociedad de Naciones (1927) o la Ville Les Terrasses, de Garches (1927). Con el paso del tiempo, Le Corbusier se irá liberando de los esquemas simétricos y recurrirá a sistemas rítmicos basados en la repetición modular uniforme.

\footnotetext{
${ }^{17}$ Le Corbusier, Hacia una arquitectura, $2^{\circ}$ Ed. Barcelona: Editorial Apóstrofe, 2006

${ }^{18}$ Ibid., p. 154

${ }^{19}$ Ibid.
} 

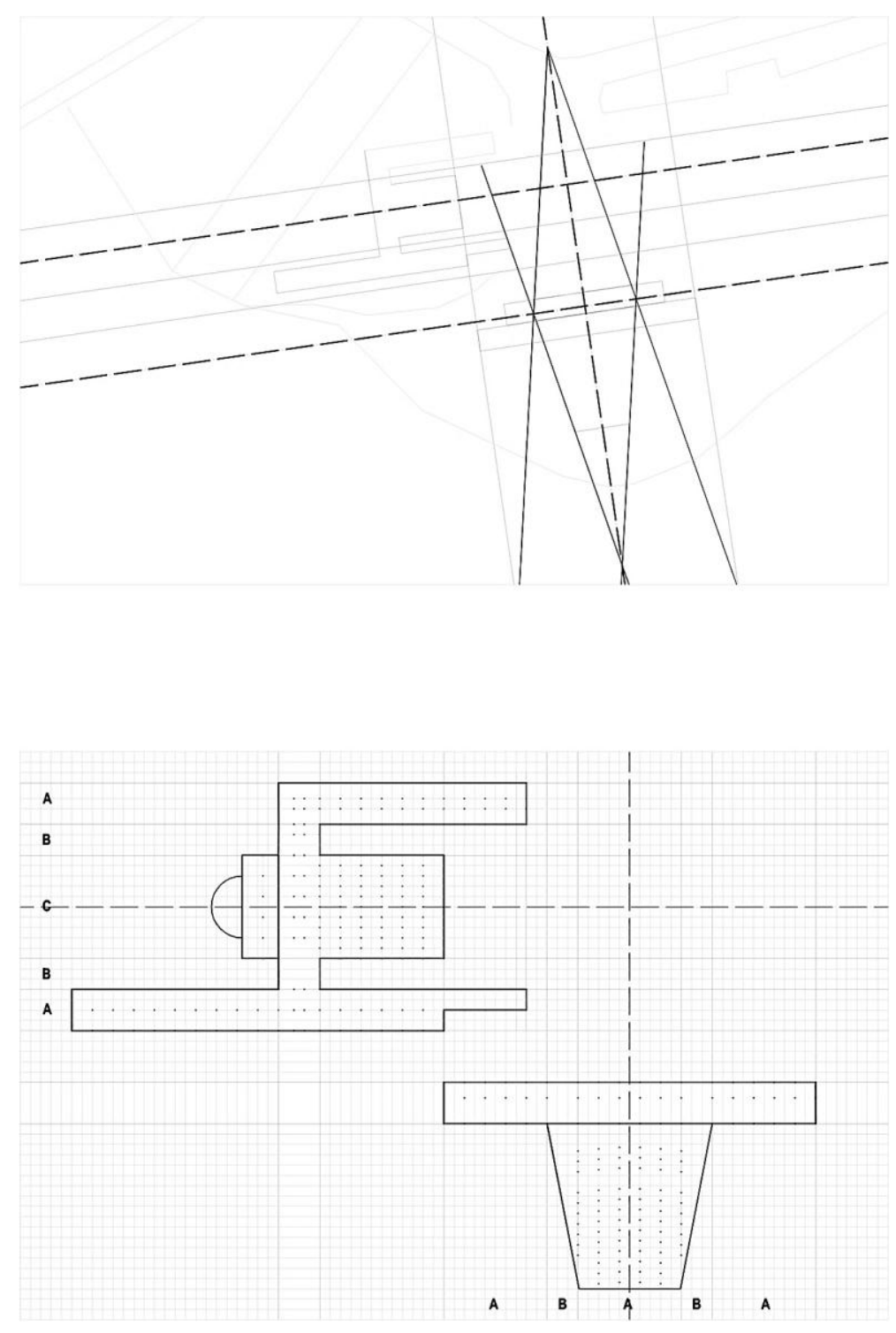

10. Palais de la Societé des Nations, Génova, 1927. Los ejes se emplean en este proyecto desde un primer momento para establecer conexiones entre la intervención y su contexto, para relacionar los volúmenes entre sí y para imprimir en el conjunto un orden jerárquico. Estos ejes a su vez determinan la dirección de la retícula, que introduce una unidad estructural en todo el complejo institucional. Esta retícula es cuadrada pero varía al coincidir con los ejes longitudinales de cada volumen, acentuando la significación de esa dirección concreta. En este proyecto, las reglas académicas de composición, como los ejes, la simetría bilateral y el ritmo ABCBA y ABABA, están muy presentes. 


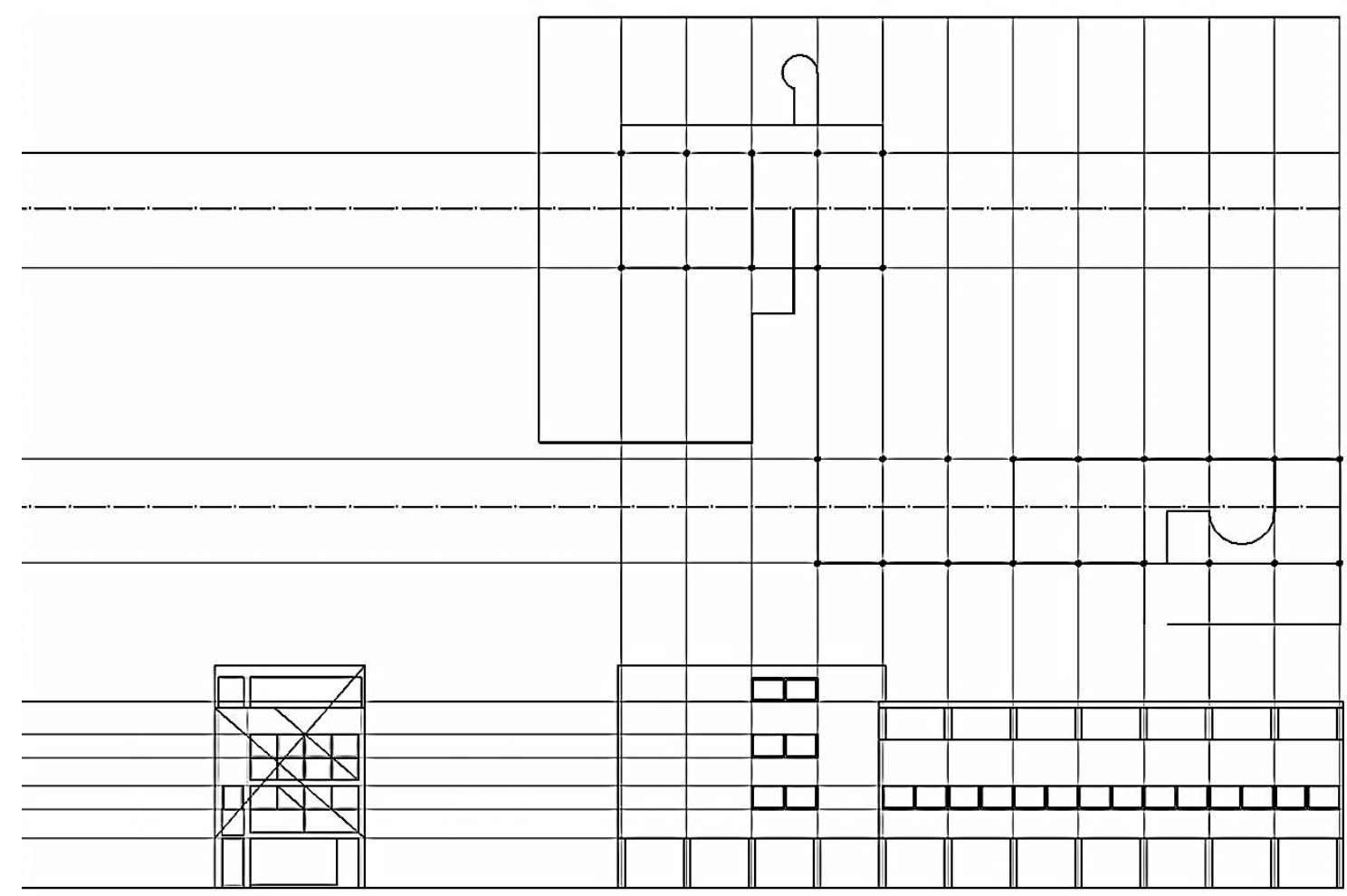

11. Weissenhof-Siedlung. Stuttgart, 1927. Una disposición rítmica de los pilares sobre un eje se empleará como estrategia que ordene el conjunto residencial y como instrumento para relacionar ambos volúmenes entre sí, dando lugar a un todo cohesionado. De nuevo, la distancia entre pilares a lo largo del eje longitudinal es de 5 metros. Los trazados reguladores empleados en la composición del alzado longitudinal combinan líneas horizontales, situadas en el "lugar del ángulo recto", y un ritmo vertical uniforme impuesto por la retícula de pilotis.

\section{Desarrollo y evolución en el empleo de las estrategias geométrico-matemáticas entre 1923 y 1933: La progresiva transparencia de las estructuras geométricas.}

Una vez clasificadas y analizadas las distintas estrategias de composición, resulta necesario dirigir una mirada global al conjunto de obras estudiadas. De este modo, al recorrer de manera transversal los distintos proyectos elaborados a lo largo de los años veinte, es posible apreciar una evolución en el empleo de las distintas estructuras geométrico matemáticas de las que se sirve Le Corbusier para componer sus proyectos. Este desarrollo coincide con la consolidación del registro formal del arquitecto -desde las obras puristas de sus primeros años, hasta el lenguaje brutalista de los años cincuenta-, por lo que su análisis puede alumbrar el estudio de la trayectoria del gran maestro del siglo XX.

En las primeras "obras blancas" de los años veinte, podemos observar que Le Corbusier, en general, se sirve de las mallas reticulares para la composición de la planta, y se apoya en el ángulo recto, el ritmo, la simetría o el número de oro para diseñar los alzados. Es decir, a diferencia de la arquitectura academicista, los planos verticales y los planos horizontales se ordenan según sus propios reglas de composición, que son distintas entre sí. El uso de la retícula ortogonal para ordenar las plantas (como en la Maison Guiette, el pabellón de L'Espirit Nouveau, la Ville Savoie, etc), vendrá determinado por las exigencias del esquema estructural porticado a base de pilotis. Mientras tanto, la fachada permanecerá indiferente al ritmo impuesto por la estructura, y se servirá de trazados de naturaleza geométrica y juegos de proporciones para ordenar los distintos huecos que en ella se dibujan, como si de una composición purista se tratase. En otros casos, el orden de la planta vendrá impuesto, no tanto por la malla estructural, sino por la forma del solar, como en las viviendas entre medianeras, la casa Fuerte 
(1925), la maison Ternisien (1926), la Ville Planeix (1927), o el ático de Beistegui (1930). En estos casos, la fachada continuará siendo objeto de una composición libre basada en el empleo del ángulo recto, el ritmo, la simetría, o el número de oro. Sólo en unos pocos proyectos, como la manufactura Frugés (1926), la Ville en Garches (1927) o los alzados longitudinales para la Weissenhoff-Siedlung (1927) la composición del alzado revela el ritmo de la planta (es decir, la distancia entre los pilotis que componen la planta coincide con el ritmo presente en los alzados). La independencia entre la composición de las plantas y los alzados, será posible gracias a la libertad que ofrecerán los nuevos materiales de construcción y el sistema estructural porticado que de ellos deriva. Pero además, el arquitecto disocia la composición de la planta del diseño del alzado al considerarlos elementos autónomos, absolutamente independientes entre sí. De este modo, el uso de distintos instrumentos de composición para las plantas y los alzados viene a subrayar dos de los cinco puntos que establece Le Corbusier para una nueva arquitectura: el plan libre y la fachada libre.
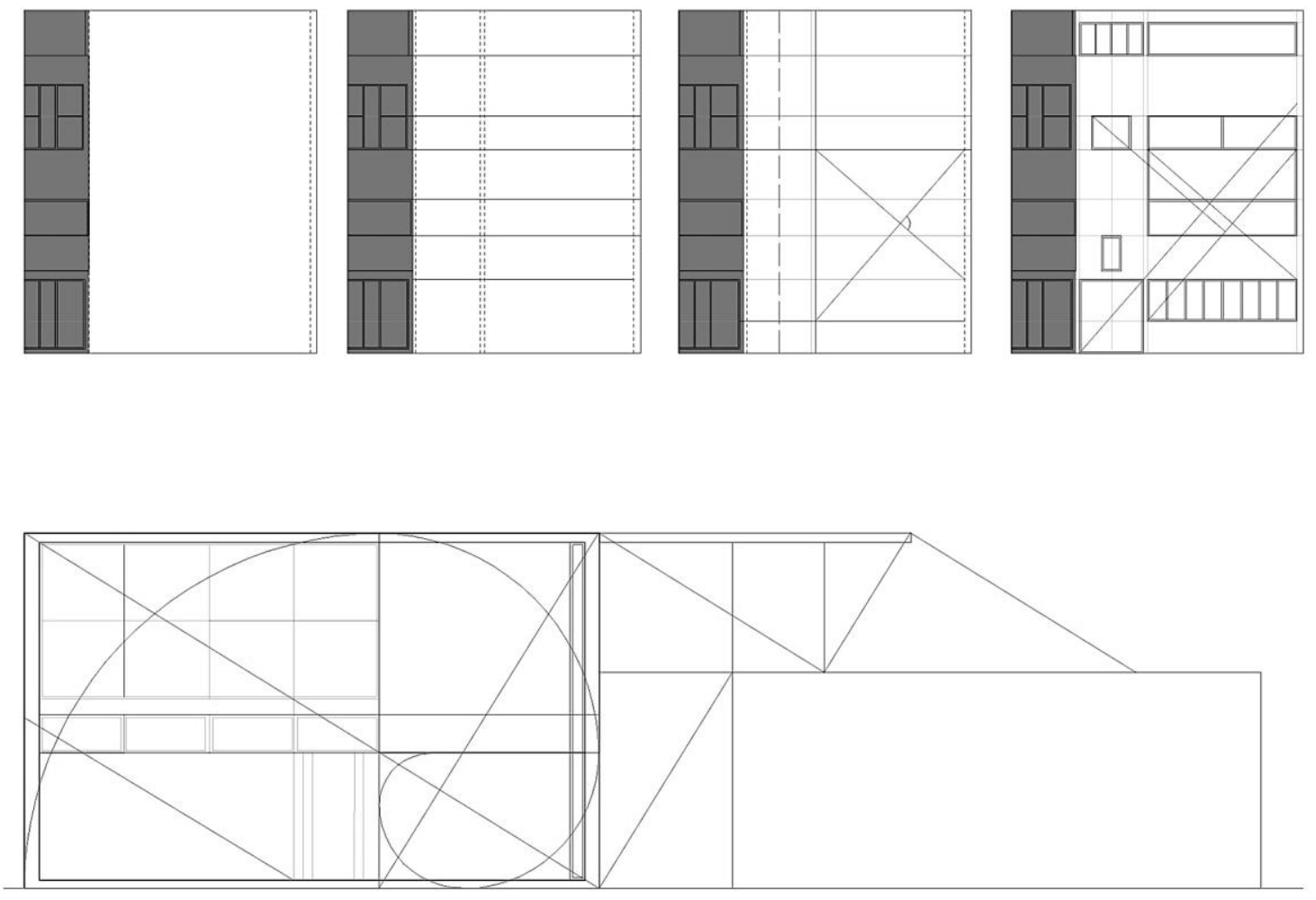

12. Casa Fuerte, París, 1925 (arriba); Maison Ternisien, Boulogne-sur-Seine, 1926 (abajo). El proyecto para la casa Fuerte se sitúa en una parcela entre medianeras de 7.60 metros de crujía con un sistema estructural de muros de carga. Fuertemente condicionada por el contexto en el que se inscribe, la solución trata de establecer una continuidad con la fachada adyacente sirviéndose, para ello, de las mismas líneas horizontales que rigen su composición. Le Corbusier divide el alzado del proyecto en dos zonas, aprovechando la colocación de un pilar estructural para salvar la luz. La parte derecha, la más próxima a la vivienda contigua, servirá para articular el cambio de escala que introduce con los grandes huecos de su composición respecto a las ventanas convencionales de la fachada preexistente. Esta parte consta de tres huecos pequeños dispuestos sobre un eje de simetría, cuya altura viene delimitada por las líneas horizontales toma como punto de partida inicial. En el resto de la fachada, Le Corbusier se sirve de unas líneas diagonales y sus perpendiculares para fijar una serie de relaciones y proporciones que determinan la anchura de los huecos y fijan composición definitiva. Por otra parte, la Maison Ternisien se sitúa en un solar complejo, y su estructura se compone de una serie de muros de carga perimetrales. No hay pilotis, por tanto, la fachada queda libre de cualquier eje estructural que respetar. La sección aurea se aplica para dimensionar los huecos de las fachadas, pero no se limita a un tratamiento superficial de las mismas, sino también da proporción a los distintos volúmenes que componen la vivienda, estableciendo una armonía de proporciones. El proyecto se convierte en un juego sabio y magnífico de volúmenes basado en el número de oro. En ambos casos, mientras el diseño de la planta se encuentra fuertemente condicionado por el solar en el que se inserta, el alzado sigue su propia lógica interna, y goza de gran libertad compositiva. 
Sin embargo, a partir de 1929, y de manera progresiva, el uso de la retícula como instrumento de composición aparece, no sólo en las plantas, sino también en las fachadas, esto es; Le Corbusier recurre a la retícula para dibujar sus alzados. En ellos, los trazados reguladores ya no serán líneas virtuales, es decir, no se tratarán de líneas invisibles que aparecen en los primeros croquis pero se borran en el objeto resultante (como sucedía en las obras puristas de sus primeros años), sino que se revelan en la misma fachada, de modo que el diagrama que rige la composición se imprime en el alzado. El primer ejemplo lo encontramos en l'Armée su Salut. En este edificio, el ritmo vertical de la retícula que compone el alzado coincide con la distancia entre pilotis de la planta, pero, sin embargo, entre el despiece del alzado y el ritmo de pilotis hay un ligero desfase sobre el eje longitudinal, de tal modo que no llegan a coincidir sobre el mismo eje. El paño acristalado de inspiración fabril se coloca como una doble piel sobre la estructura del edificio y es independiente de esta, es decir, la fachada continúa siendo una fachada de composición libre.

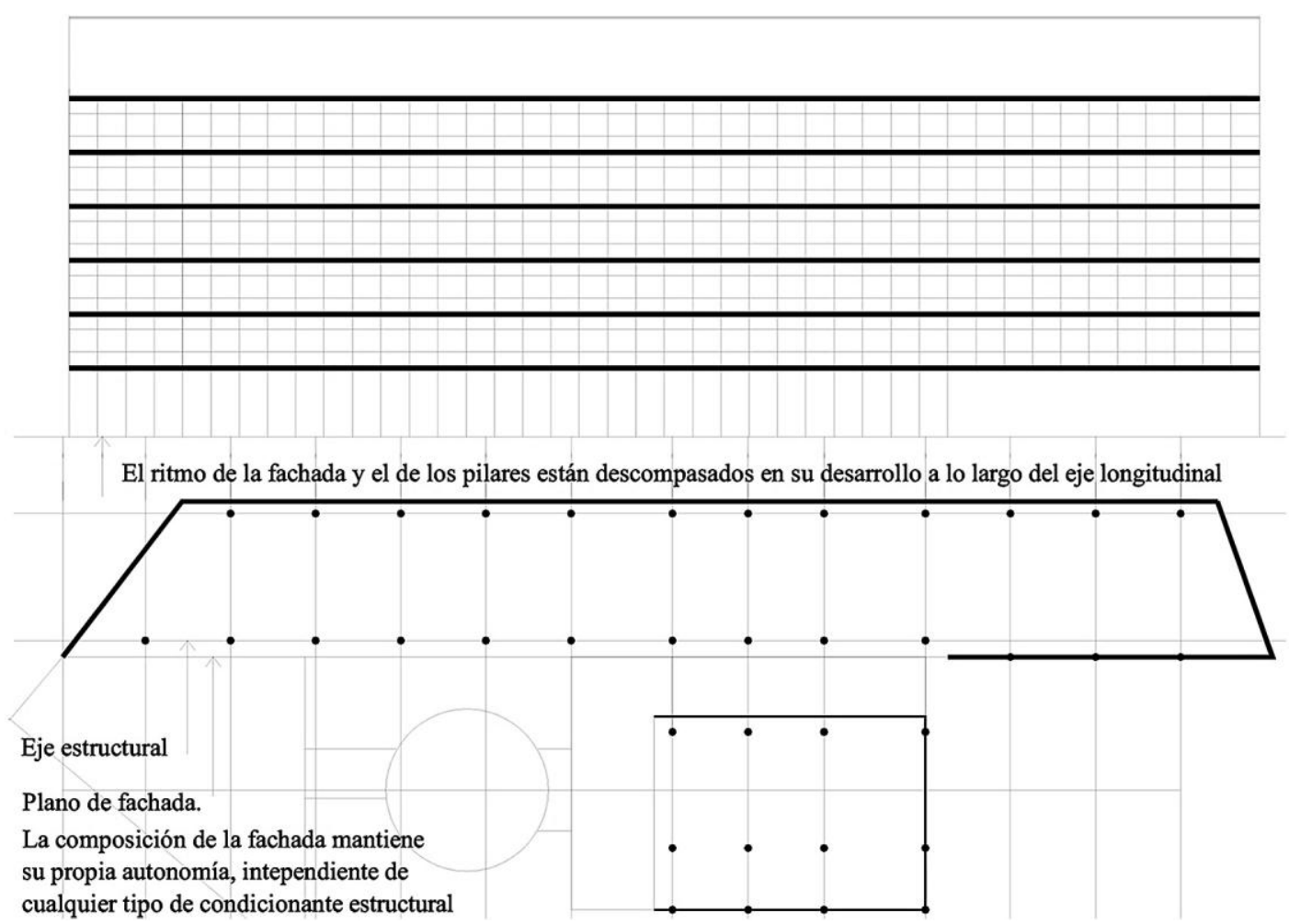

13. Armé du Salut. Cité de Refuge, París, 1929. La fachada se presenta subdividida según un módulo cuya longitud es igual a un tercio de la distancia entre pilares. El alzado principal consta de una piel de vidrio que envuelve el volumen, y que se encuentra separada de la estructura, por tanto, está exenta de cualquier condicionante impuesto por el ritmo de la estructura que pudiera condicionar su composición. Este hecho se acentúa por el hecho de que es posible apreciar una discordancia a lo largo del eje longitudinal entre el ritmo de los pilares, por una parte, y el correspondiente al plano de la fachada, por otra. En l'Armé du Salut, la composición de la fachada se rige según su propia lógica, al igual que sucedía con las obras blancas de los primeros años: sigue siendo una fachada libre. Por otra parte, este ejemplo resulta significativo porque se trata del primer proyecto en el que Le Corbusier aplica la retícula cuadriculada para regular sus alzados, y porque, por primera vez, la línea de forjados se revela en el alzado, siendo empleada como directriz horizontal que determina la composición.

Poco a poco, la estructura irá adquiriendo mayor presencia en fachada, dando cohesión y confiriendo unidad al conjunto. En la Imprimerie Draeger, la planta y el alzado ya no serán dos entidades separadas y ajenas entre sí, sino que el ritmo que ordena la planta, servirá a su vez para componer y ordenar los alzados, de modo que la retícula presente en el alzado revela el sistema porticado a base de vigas y pilares. Finalmente, en el Pabellón Suizo de la Ciudad Universitaria de París, el entramado estructural se convierte en instrumento de composición 
en sí mismo. Los planos horizontales y verticales del proyecto se regirán por las mismas leyes compositivas, que ya no son líneas virtuales de composición, ni diagramas esquemáticos, sino que adquieren corporeidad y se construyen, introduciendo un cambio de registro formal en la trayectoria de gran maestro del siglo XX.

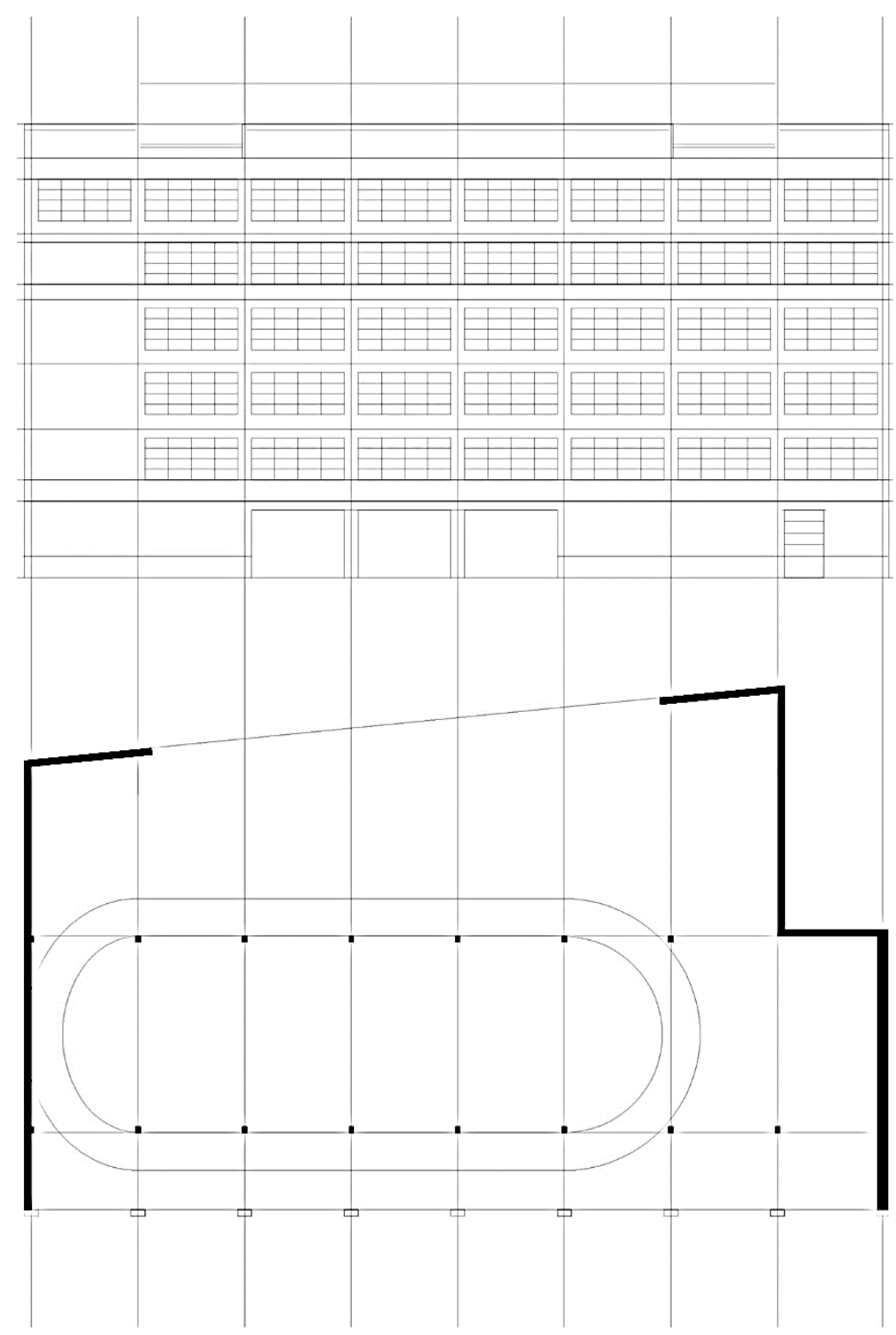

14. Imprimerie Draeger. París, 1929. En este proyecto, por primera, vez Le Corbusier revela en fachada la estructura de pilares y forjados del interior del proyecto. El ritmo de los pilares, y la distancia horizontal entre forjados se traduce en una malla ortogonal con la que el maestro suizo compone los alzados de la Imprimerie. La estructura imprime un ritmo modular vertical (determinado por las directrices de los pilares) y una división horizontal (condicionada por las distancias entre forjados). Se trata del primer proyecto donde la composición de la fachada no es independiente al orden interno del proyecto, sino que depende de la estructura, la fachada deja de ser una máscara. 

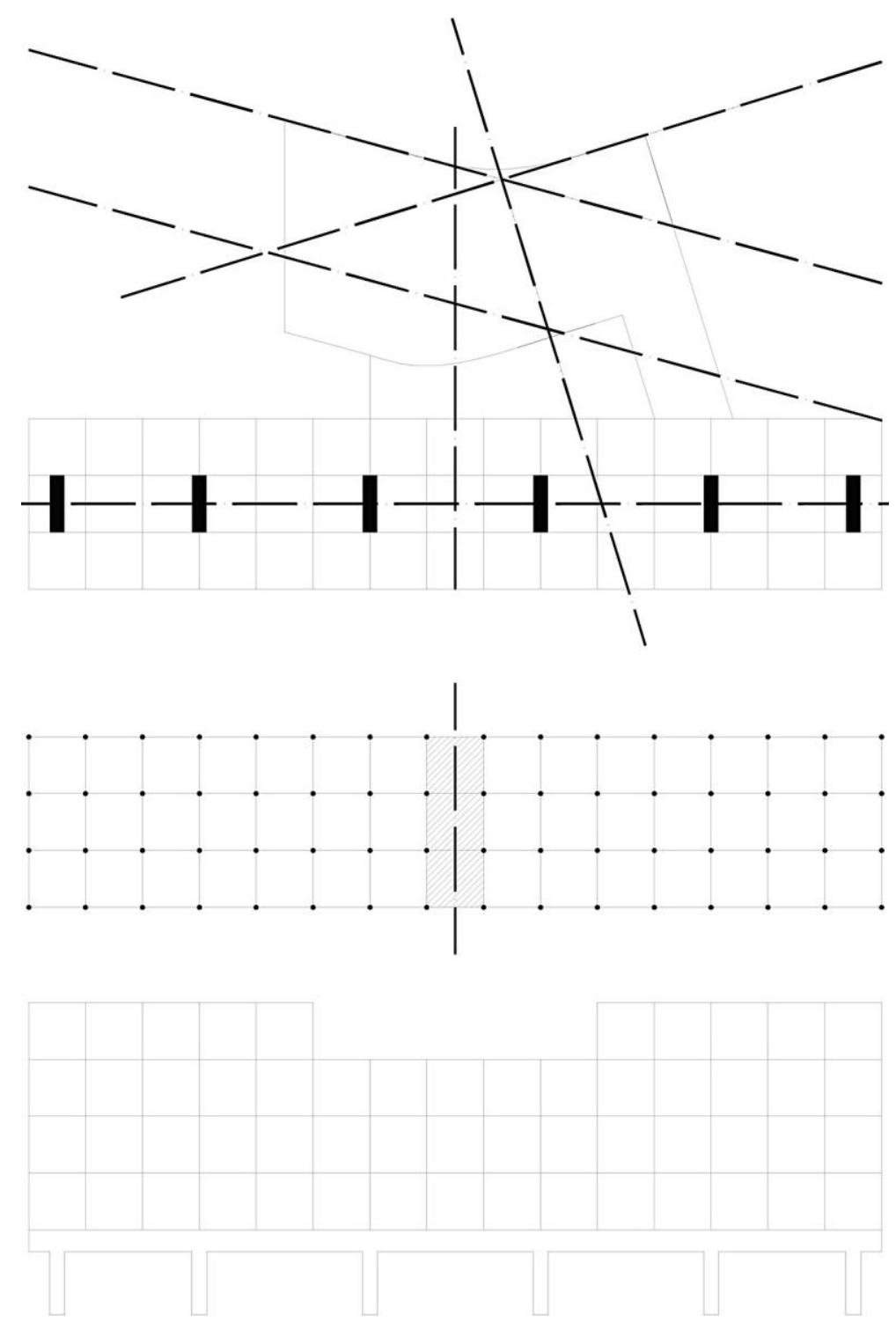

15. Pabellón Suizo, Ciudad Universitaria. París, 1930. El sistema estructural empleado (un entramado ortogonal formado por elementos longitudinales, iguales entre sí) deriva en un trazado regulador. Se trata de un sistema geométrico tridimensional que imprime su orden a todo el proyecto, determinando las reglas de composición que deben regir la composición de la fachada, la distribución de la planta, y la proporción de sus secciones internas; los trazados reguladores ya no más se aplican sobre superficies planas, ya no se trata de una composición pictórica cubista; la propia construcción dibuja la imagen del proyecto, inaugurando, con ello, una nueva etapa en la producción arquitectónica de Le Corbusier.

\section{Bibliografía.}

Baker, Geoffrey H. Le Corbusier, Análisis de la forma, Barcelona: Gustavo Gili, 1985

Le Corbusier, Le Corbusier Archive, New York: Garland Publishing, 1984

Le Corbusier, Une maison, un palais: "a la recherche d'une unité architecturale”, París: G.Cres, 1928

Le Corbusier, El Modulor, Barcelona: Editorial Poseidón, 1976

Le Corbusier, Hacia una arquitectura, $2^{\circ}$ Ed. Barcelona: Editorial Apóstrofe, 2006

Summerson, John, El lenguaje clásico de la arquitectura, 11ª Ed. Barcelona: Editorial Gustavo Gili, 1998 